\title{
Por una antología de reseñas y otras prácticas por-venir
}

ynalía Gerbaudo / Universidad Nacional del Litoral - Conicet

agerbaudo@fhuc.unl.edu.ar; analia.gerbaudo@conicet.gov.ar

Comme Kafka, il faut viser la petite tache plutôt que le spectaculaire, il faut réduire l'échelle de l'enquête ou de l'action (...). Une configuration globalisée exige différents types de raids régionaux et un art plus subtil du pronostic.

Avital Ronell, Entretien

Durante el último Congreso Internacional Cuestiones Críticas celebrado en la Universidad Nacional de Rosario en 2015 los organizadores promovieron un panel específicamente destinado a discutir el problema planteado por El taco en la brea en la editorial del número anterior y multiplicado en publicaciones y redes desde las posiciones más diversas. ${ }^{1}$ La mesa reunió, además de los expositores convocados (entre ellos, Jeffrey Cedeńo y Alberto Giordano con la coordinación de Laura Utrera), a investigadores, profesores, becarios y estudiantes de posgrado de diferentes regiones de Argentina y de América Latina preocupados por el sentido de nuestras prácticas de escritura frente a los parámetros globales de institucionalización e internacionalización más o menos estandarizados. Y quisiera insistir en este matiz ya que la encendida caracterización de cómo este problema se cristaliza en diferentes disciplinas, instituciones y países no sólo visibiliza una grieta para la discusión sino también para la acción política entendida en la productiva dirección en que Eduardo Rinesi la ha pensado: ante la falta y el descontento con un estado de las cosas, se aprovechan las fisuras del «orden» (23) para inventar otras posibilidades.

Es en esta vía y alrededor del problema sobre el que vengo rondando que quisiera comunicar un conjunto de decisiones tomadas por quienes hacemos El taco en la brea incluida la reposición en la sección «Archivo» de este número de dos textos que lo rozan mientras abren otras dimensiones de análisis.

La primera decisión se desprende de la convocatoria deslizada en nuestra editorial del número anterior: atenta lectora, Mónica Cragnolini avaló la propuesta de componer un dossier con el título «¿Para quién escribimos?». Entusiasta, Isabel 
Quintana se ofreció a coordinarlo y Rafael Arce a publicar en este tercer número el que preparaba junto a Soledad Nívoli sobre «El retorno de la imaginación». Dado este marco y el interés que advertimos en el campo por discutir estas cuestiones, ampliamos la convocatoria inicial a trabajos para el dossier «¿Para quién escribimos?» en dos sentidos: por un lado, invitamos a escribir a investigadores que puedan aportar resultados sobre este tema, integren o no el Comité Científico de nuestra revista; por el otro, extendemos el plazo de recepción de estos artículos hasta el 30 de junio de 2016.

Esta decisión se encadena con otra: por protección de quienes confían los resultados de sus investigaciones a esta publicación, nos ajustamos a los parámetros de evaluación seguidos para la indexación de las revistas científicas en el punto relativo a la frecuencia semestral. Contar con un número en mayo y otro en noviembre, por un lado, agilizará la circulación de los trabajos; por el otro, demanda ajustes internos en diferentes órdenes a los efectos de asegurar una cuidada articulación entre quienes asumimos responsabilidades en la gestión de la publicación que además, implementa el Open Journal System con sus ya muy reglados protocolos. Así es como, a la elaboración de un «Manual de procedimiento» para regular el trabajo de gestión editorial interno (básicamente, el que realizan la directora, el codirector, el editor adjunto, el secretario de redacción y el comité editorial) siguió la actualización de los equipos. En esta línea quiero agradecer especialmente a todos quienes apuestan a este proyecto, tanto a los que continúan con sus roles como a los nuevos integrantes del comité editorial (Mariana Catalin, Alejandro Gasel, Luciana Martínez, Gabriela Milone, Cristian Molina y Carolina Rolle), del comité científico (Gloria Chicote, Álvaro Fernández Bravo, Ana Teresa Martínez y Gustavo Verdesio) y al Editor Adjunto, Santiago Venturini que se incorpora en este número. La lógica de armado de los comités busca solidificar un principio de acción: creemos que sólo a partir del aporte de diferentes agentes que escriben desde diferentes instituciones y desde diferentes campos y posiciones resulta posible, en especial a partir de la confrontación, enriquecer la propia perspectiva (discutir con otros obliga a afinar los argumentos que apoyan una tesis, un punto de vista). Una confrontación que no se tramita desde la perspectiva de la tolerancia sino desde el diálogo (en el poco condescendiente sentido bajtiniano) y el debate de ideas.

La tercera decisión: participamos de Latinoamericana. Asociación de revistas académicas en Humanidades y Ciencias Sociales. ${ }^{2}$ Un espacio en el que la discusión eventual propuesta para el dossier "¿Para quién escribimos?» constituye el eje constante de una conversación ampliada que nuclea hoy ya a ciento sesenta publicaciones periódicas de dos campos cada vez más atravesados por el re-uso creativo y el intercambio de conceptos, protocolos y metodologías (el listado de trabajos que probarían esta productiva «contaminación» sería enorme; sólo por citar algunos nombres, a los clásicos de Beatriz Sarlo, Horacio González, Raúl Antelo y Mónica Cragnolini, se suman los más recientes de Rossana Nofal, Julieta Yelin y Evelyn Galiazo y dos tesis doctorales en proceso de defensa mientras 
redacto esta presentación: una, de impactante osadía metodológica —Daona-; la otra, de im-posible delimitación entre ensayo filosófico/literario -Billi-).

En esa misma línea, suscribimos al documento elaborado el 29 de setiembre de 2014 en Santiago de Chile durante la reunión de editores de publicaciones periódicas latinoamericanas realizada por iniciativa de la Revista chilena de literatura con el objetivo de discutir políticas conjuntas. Tal como fue acordado, publicamos el texto entonces resultante con la actualización del listado de revistas adherentes que llega a la fecha de cierre de este número de El taco en la brea prácticamente a la centena.

Como se advertirá, desde mayo de 2015 hasta el presente discutimos el problema entonces bosquejado en la editorial del número 2 en todos los sitios que estuvieron a nuestro alcance.

Pero por otro lado, en una notable actuación del punto de vista que defienden, tres investigadores se han ocupado del tema desde muy recientes publicaciones en formato libro editado en papel (punto de vista que promueve la lógica del «y... у...» por contraste con la del «o... o...»: no se trata de una disyuntiva entre el libro o el artículo, el soporte papel o el digital). En las antologías de ensayos organizadas por Alberto Giordano y por Clara María Parra Triana y Raúl Rodríguez Freire se advierte tanto la misma preocupación por el sentido del trabajo crítico como la asunción del colectivo desde el que se lo tramita. Recortada sobre América Latina, Parra Triana y Rodríguez Freire realizan una selección orientada por la fantasía de incidir sobre el canon crítico y teórico continental a partir de la reposición de textos clásicos de la teoría y la crítica presentados en una estructura singular: cada uno es acompañado por una breve nota firmada por otro crítico que justifica por qué es necesaria hoy su relectura. Recortada sobre la Argentina de la los ochenta y la posdictadura, ${ }^{3}$ Giordano recoge ensayos firmados por «especialistas» (y se apura en aclarar: "ninguno de ellos aceptaría que se lo distinga de este modo, aunque así es como los reconoce la comunidad a la que pertenecen») que caracteriza como «empeńados en la transmisión problematizadora de saberes sobre las humanidades y las ciencias sociales en contextos universitarios» (II). Contextos sobre los que busca incidir retomando un tipo de producción situada «en los márgenes de la cultura que legitima el vínculo reproductivo entre investigación y escritura»: "la figura inspiradora del "maestro ignorante" y la moral emancipatoria de la igualdad de las inteligencias (Rancière) no sobreviven la institucionalización» (8), pareciera concluir. Sin embargo más adelante, mientras destaca en el "ethos del recurso al ensayo» un "estilo de vida académica, inconforme y disidente» (I2) repasa, desde la posición de un «lector apasionado», los avatares que el género logró sortear una vez asumidos «los riesgos de institucionalizarse» (I5). Tarea que logra con relativo éxito tal como lo demuestra (como Giordano reconoce) el financiamiento de la práctica (tanto por agencias «literario-artísticas» como científicas) difundida además en la enseñanza de grado y de posgrado y promovida desde la gestión de la investigación que avala proyectos dedicados recursivamente a analizarla. 
No fue esa la suerte que ha corrido otro género que sólo en contados casos ha podido asociarse a una posición de inconformismo o disidencia intelectual dentro del campo: la reseña. Casos entre los que, en la zona de borde entre ciencias humanas y sociales desde la que trabajamos, descolla la revista Los libros de la que también se ocupaba Jorge Panesi en la mítica conferencia «La caja de herramientas o qué no hacer con la teoría literaria» (1996). En aquella ocasión destacaba el gesto de resistencia contra-institucional tramado durante la dictadura que se había iniciado con Onganía desde una formación que, agrego, nacía básicamente como una revista de reseńas. Pocas veces el género logró tal nivel de exigencia y de mordacidad. Y si bien es necesario reconocer que en algunas pocas ocasiones rayó el adjetivo banal (cf. Panesi 1985:42), dominaba un estado de alerta respecto del vaciamiento de la crítica contra el que explícitamente batallaban (cf. Rosa). Vaciamiento que se advierte en el tipo de reseña pensada como una invitación a tomar el té a las cinco de la tarde: una previsible y almibarada cantata de las bondades de un libro sin otro contenido que la repetición más o menos parafraseada de su índice que da como resultado un texto soso y prescindible que, con mucho viento a favor, logró informar sobre la novedad editorial antes de pasar al más rotundo olvido.

La publicación de las antologías de Giordano, Parra Triana y Rodríguez Freire, las discusiones generadas a propósito del sentido de nuestra escritura en publicaciones periódicas, mesas de congresos y asociaciones propician que destaquemos también esta arista del problema mencionado muy sucintamente en la editorial del número 2 de El taco en la brea. Si sólo tomo el caso de Los libros, no es para cerrar el debate o proponer un modelo. Por el contrario: si se miran con detalle las firmas reunidas tanto en la compilación de Parra Triana y Rodríguez Freire como en la de Giordano se podrá advertir la variedad de tonos, posiciones y asunciones teóricas respecto del «ensayo» por profesores universitarios e investigadores que en muchos casos, sólo pueden caer juntos en esos índices. Así, con la misma marca de heterogeneidad, imagino una antología de reseñas que, obviamente, no seleccionaría las que se escribieron limitándose a detallar, con más formalismo que convicción, de qué iba el libro. Imagino una antología que retome aquellas en las que, además de ese punteo elemental, se haya expuesto una conjetura generadora de líneas de exploración críticas y/o categoriales («junto con» o «a partir de» las que se derivaban del libro en cuestión). También desde la posición de «lectora apasionada» (es decir, también ajena a la "exhaustividad») imagino que entre las infaltables de ese libro por-venir ${ }^{4}$ estarían las que han firmado María Teresa Gramuglio sobre Cicatrices de Juan José Saer (1969) y Ema, la cautiva de César Aira (1982), Beatriz Sarlo (1996) sobre la Obra completa de Juan L. Ortiz, Miguel Dalmaroni sobre El imperio realista de María Teresa Gramuglio, Alberto Giordano (I999) sobre Las letras de Borges y otros ensayos de Sylvia Molloy. Más allá de la viabilidad o no de esta fantasía, importa escribirla por los interrogantes que pretendemos suscitar: tenemos una responsabilidad que se traduce en prácticas cada vez que discutimos desde qué parámetros aceptamos o rechazamos una reseña en una 
publicación periódica (¿deben pasar por referato?, ¿sólo por el comité editorial y el control del editor adjunto y el secretario de redacción?, ¿desde qué criterio se las evalúa?, etc.) y luego decidimos; cada vez que formamos a un estudiante en los protocolos de este género (comparto la preocupación de Daniel Link cuando encuentra reseñas de alta potencia crítica y heurística firmadas por un estudiante: ¿Cómo se recibe en el campo que un/a «recienvenido/a», como diría Macedonio, exponga una hipótesis que confronte con puntos de vista hegemónicos y/o enunciados desde una posición dominante?, ¿cómo se reacciona cuando ese/a «recién llegado/a» hace emerger debates hasta entonces subterráneos mientras corre todos los riesgos del trabajo intelectual, reñido con el miedo a la exposición?); cada vez que defendemos en una comisión de gestión de la investigación una postura a favor o en contra de su consideración al momento de "puntuar» para obtener una beca o un subsidio; cada vez que optamos por invertir tiempo en su escritura o delegarla a «los recursos humanos en formación».

Finalmente, respecto de la responsabilidad traducida en prácticas con sus consecuencias en el trabajo (y por lo tanto en la vida) de los otros dados los «efectos de campo» de ciertas decisiones de gestión institucional tomadas desde una posición dominante, en este número se rescatan desde la sección «Archivo» dos textos ligados a dos discusiones fundamentales: por un lado, una vuelta reflexiva sobre el sentido y el futuro de nuestros posgrados. Un diagnóstico centrado en especial en Argentina y en Brasil que Raúl Antelo presenta en 2013 a propósito de un Seminario dictado en la Universidad Nacional de Cuyo en Mendoza y que, como es usual en sus trabajos, expande la pregunta nodal para llevarla, en este caso, a interrogar el futuro de la investigación literaria continental y sus reales posibilidades de internacionalización (en particular cuando se declinan en portugués o en espańol). Por el otro, una brevísima intervención realizada justamente en español y vía teleconferencia por Ariel Wilkis desde Buenos Aires a propósito de la celebración de los 40 años de la École des Hautes Études en Sciences Sociales en 20I5. Un texto escuchado en París sin traducción cuyas potentes anécdotas solicitan nuestro lugar (como argentinos, como latinoamericanos y, otra vez, como hablantes de español, lengua en la que además circula la mayor parte de nuestra producción) en la escena teórica internacional (no es un dato menor que después de su lectura un profesor parisino le preguntara entusiasmado a Wilkis sobre los usos de Althusser en Argentina: una inquietud que si bien reconoce la creatividad en la reapropiación, se inserta en el marco general de un desconocimiento de la producción teórica latinoamericana y de un interés hegemónico —en el nunca totalizador sentido williamsiano- por la circulación Norte-Sur). Un tema sobre el que se volverá en el próximo número de El taco en la brea y que se despunta aquí a partir de estos dos textos que merecen ampliar sus destinatarios iniciales y sobrepasar la fuga que impone la oralidad dado el conjunto de preguntas que plantean y las prácticas que, con seguridad, estimularán. ${ }^{5}$ Dos textos necesarios para los debates por-venir. 


\section{Notas}

${ }^{1}$ Entre otros ítems para el análisis, destaco el que despunta Juan Pablo Alperín en un artículo que señala la contradicción entre, por una parte, el financiamiento a la investigación casi exclusivamente en los países «en desarrollo» por los gobiernos a través de agencias nacionales unida a la publicación de "una parte importante de los resultados» en «revistas académicas locales y regionales» $y$, por la otra, el uso de indicadores de evaluación de la producción e impacto tomados de los «llamados índices "internacionales" (principalmente la Web of Science - WoS - de Thompson-Reuters y Scopus de Elsevier) donde sólo se incluye una pequeña fracción de las revistas académicas de regiones en desarrollo» (I6). Su trabajo, además de interpelar a crear otros indicadores (y de proponer algunos) permite retomar la discusión sobre el diseminado término «internacionalización» y las pretensiones nunca desinteresadas de su definición unívoca (el énfasis en las métricas alternativas tiene su correlato con las prácticas de los investigadores del campo literario que en Argentina, frente a la presión por publicar papers en revistas «tipo I», privilegian el libro —impreso-, los blogs y/o Facebook —cf. Gerbaudo 2015a, 2015c, 2015c-).En esta misma línea cabe repasar las diez sugerencias contenidas en el Manifiesto de Leiden (Hicks y otros) y su crítica al privilegio de la perspectiva cuantitativa al momento de evaluar una producción, al desconocimiento de las tradiciones regionales y de las diferencias por disciplina.

Para una crítica a la «cultura de indicadores tecnocráticos», ver Santos, Subercaseaux, Rojo, Villalobos, Tejeda. Entre las publicaciones recientes que se han ocupado de este aspecto del tema cabe mencionar el muy controversial número monográfico de la revista $L i$ teratura: teoría, historia, critica, "Las humanidades y los estudios literarios en la era de la excelencia académica» (cf. Díaz Villareal). Para el análisis de la relación entre mercado y tecnocratización de los procesos de evaluación de la investigación, ver los incisivos y necesarios trabajos de Adrián Freja, Raúl Rodríguez Freire (2012, 20I5) y Maximiliano Tello (20I2). Para un diagnóstico sobre prácticas de los investigadores en ciencias sociales y humanas en Argentina, ver Beigel (2014, 20I5) junto a Gallardo (20I4a, 20I4b) y Salatino (20I4). Finalmente en el marco de un trabajo grupal estamos realizando un diagnóstico sobre las prácticas de los investigadores del campo de las letras en Argentina entre 1945 y 2015 (los instrumentos construidos para este trabajo y sus actualizaciones - ampliación de la muestra, ajuste de criterios, etc. - pueden consultarse en Gerbaudo 2015b, 2015d).

${ }^{2}$ Para los fines y actividades de esta Asociación, incluida la actualización constante de instituciones participantes, ver http://revistaslatinoamericanas.org/

${ }^{3}$ En trabajos previos leímos el período recortado como «posdictadura» entre 1984 y 2003 por las razones que allí explicitamos (Gerbaudo 20I2, 20I5e; cf. Antelo). La mayor parte de las medidas tomadas por Mauricio Macri desde su asunción como presidente de la nación en diciembre de 2015 en los planos económico, educativo, cultural, jurídico, etc., exige una reconceptualización ya que los retrocesos en estos planos reactualizan, desde una pretendida cuidada clave comunicacional «post» ("postpopulismo», "postnacionalismo", "postpolítica»), posiciones defendidas durante el «Proceso de reorganización nacional» (sobre por qué volver sobre este modo de nombrar, ver en este mismo número Schwarzböck). Estos retrocesos activan lo que en esos mismos trabajos afirmamos respecto de la fragilidad de todo augurio de "fin de», del poroso borde que debilita las demarcaciones y del carácter siempre por-venir de la democracia (cf. Derrida 1991, 1993).

${ }^{4}$ Cuando hablo del género «reseña» incluyo también los textos armados para presentaciones de libros que en muchas ocasiones, terminan transformándose en reseñas. En líneas generales, siguen los mismos protocolos (con las traducciones propias requeridas por el pasaje del registro oral al escrito).

${ }^{5}$ En Creencia y acontecimiento. El sujeto después de la teoría, Marcelo Topuzián pone en serie la producción teórica de Jacques Derrida, Slavoj Zizek, Alain Badiou y Ernesto Laclau. La inclusión de Laclau en esta serie abre un interesante caso para el análisis: ¿̇se reconocería su estatuto teórico si no hubiera desarrollado su trayectoria desde la Universidad de Essex y publicado buena parte de su producción en inglés (con el efecto de campo que 
genera, además, enunciar desde el polo institucional citado)? La pregunta, se sabe, es retórica, y en su respuesta se juega no sólo un problema de lenguas sino en especial del «efecto de campo» (Bourdieu 23) que genera enunciar desde una institución ubicada en una posición dominante en el circuito de circulación internacional de las ideas.

\section{Bibliografía}

Alperín, Juan Pablo (20I4). «Indicadores de Acceso Abierto. Evaluando el crecimiento y uso de los recursos de Acceso Abierto de regiones en desarrollo», en Juan Pablo Alperin y otros, editores. Indicadores de acceso abierto y comunicaciones académicas en América Latina. Buenos Aires: CLACSO, I5-83.

Antelo, Raúl (20I3 [20I6]). «Programa para un posgrado futuro». El taco en la brea 3. En este mismo número.

Beigel, Fernanda (20I4). "Publishing from the Periphery: Structural heterogeneity and segmented circuits. The evaluation of scientific publications for tenure in Argentina's CONICET». Current Sociology 62, 743-765.

(2015) «Científicos "Calibanes” I: Estilos de publicación científica, capital lingüístico y circuitos de consagración en Argentina. Un análisis de las publicaciones "más relevantes" de los investigadores del CONICET». Coloquio Social Sciences and Humanities in the Changing North-South Relations. Córdoba: unc/InTERCo SsH.

Beigel, Fernanda y Osvaldo Gallardo (20I4a). «La estructura del campo científicouniversitario argentino: expansión de las capacidades de investigación, heterogeneidad estructural y circuitos segmentados». Informe $\mathrm{N}^{\circ}$ I. Mendoza: PIDAAL/INTERCO SSH.

(20I4b). "Potencial, fortalezas y debilidades de las ciencias sociales y humanas en Argentinas: Los/las investigadoras/es». Informe $\mathrm{N}^{\mathrm{o}}$ 2. Mendoza: PIDAAL/INTERCO SSH.

Beigel, Fernanda y Maximiliano Salatino (20I4). "Potencial, fortalezas y debilidades de las ciencias sociales y humanas en Argentinas: las revistas». Informe $\mathrm{N}^{\mathrm{o}}$ 3. Mendoza: PIDAAL/ INTERCO SSH.

Billi, Noelia (2015). Modos de morir. Impersonalidad, materialismo y constitución de la subjetividad en la obra de Maurice Blanchot. Tesis de Doctorado de la Universidad de Buenos Aires (Filosofía). Buenos Aires: Universidad de Buenos Aires.

Bourdieu, Pierre (200I). Science de la science et réflexivité. Cours du Collège de France 2000-200I. París: Raisons d'agir.

Dalmaroni, Miguel (2002). «El imperativo realista y sus destiempos». Anclajes 6, 44I-468.

DaOna, Victoria (2015). Las voces de la memoria en la novela argentina contemporánea: militantes, testigos e hijos/as de desaparecidos/as (2000-2014). Tesis de Doctorado en Ciencias Sociales. Los Polvorines/Buenos Aires: Universidad Nacional de General Sarmiento/Instituto de Desarrollo Económico y Social.

Derrida, Jacques (I99I). L'autre cap. La démocratie ajournée. París: Minuit.

(1993). Spectres de Marx. L'État de la dette, le travail du deuil et la nouvelle Internationale. París: Galilée.

Díaz Villarreal, William (2015). "Las humanidades, la universidad y la era de la excelencia académica (a manera de introducción)». Literatura: teoria, historia, crítica 17, 19-38. 
Freija, Adrián (20I5). «Editorial». La palabra 27, 9-II.

Gerbaudo, Analía (2012). «Sobre la dicha de tener polémicas». Estudios de Teoría Literaria 2, 83-98 [en línea]. Consultado el I4 de julio de 2015 en http://fh.mdp.edu.ar/revistas/index. $\mathrm{php} / \mathrm{et} /$ issue/current

- (2015a). «Algunos episodios alrededor de la institucionalización y la internacionalización de la investigación literaria en la Argentina de la posdictadura (y después)». IV Congreso Internacional Cuestiones Críticas. Rosario: UNR (en prensa).

(20I5b). «Annexes (Deuxième rapport technique)». Linstitutionnalisation des études littéraires à l'université argentine (1945-2010) [en línea]. Santa Fe: UNL, 6I-72. Consultado el I6 de diciembre de 2015 en http://www.fhuc.unl.edu.ar/media/investigacion/centros/ cedintel_oct_I5.pdf

(2015c). «Una pregunta fundamental».Panel «Los problemas de la gestión editorial: las revistas de la crítica literaria y cultural en América Latina». IV Congreso Internacional Cuestiones Críticas. Rosario: UnR.

(2015d). «Binomios, controversias y algunos episodios alrededor de la internacionalización de la investigación literaria argentina». Tercer Coloquio de avances de investigaciones del CEDINTEL. Santa Fe: UNL. En prensa.

(2015e). Politicas de exhumación. Las clases de los críticos en la universidad argentina de la posdictadura (1984-1986). Los Polvorines/Santa Fe: UnGs/UnL, 2016.

Giordano, Alberto (1999). «Lo novelesco de la crítica. Las letras de Borges de Sylvia Molloy». Modos del ensayo. De Borges a Piglia. Rosario: Beatriz Viterbo, 267-276.

(Ed.) (2015). El discurso sobre el ensayo en la cultura argentina desde los 80. Buenos Aires: Santiago Arcos.

Gramuglio, María Teresa (1969). «Las aventuras del orden. Juan José Saer. Cicatrices». Los libros 3, 5-24.

(1982). «Increíbles aventuras de una nieta de la cautiva». Punto de vista I4, 27-28.

Hicks, Diana y otros (2015). «The Leiden Manifiesto por Research Metrics». Nature 520, 429-43I.

Panesi, Jorge (1985). «La crítica argentina y el discurso de la dependencia». Críticas. Buenos Aires: Norma, 2000, 17-48.

(1996 [2014]). «La caja de herramientas o qué no hacer con la teoría literaria». El taco en la brea I [en línea], 322-333. Consultado el 7 de febrero de 2016 en http://www.fhuc. unl.edu.ar/centros/cedintel/eltacoenlabreaor_2or4.pdf

Parra Triana, Clara María y Raúl Rodríguez Freire (Comp.) (20I5). Crítica literaria y teoría cultural en América Latina. Para una antología del siglo XX. Valparaíso: Ediciones Universitarias de Valparaíso.

Rinesi, Eduardo (2003). Politica y tragedia. Hamlet entre Hobbes y Maquiavelo. Buenos Aires: Colihue.

Rodríguez Freire, Raúl (2OI2). «Arte, trabajo, universidad», en Iván Pincheira Torres, coordinador. Archivos de frontera: el gobierno de las emociones en Argentina y Chile del presente. Santiago de Chile: Escaparate, I6I-I9I.

- (2015). "La universidad "de calidad" y las ruinas del pensamiento» (entrevista de Nelly Richard). The Clinic 628, 26-28. 
Rojo, GrínOr (2013). «Estropicios académicos y tanteos críticos». Revista chilena de literatura 84, I29-I43.

Rosa, Nicolás (1972). «Contracrítica». Los Libros 28, 2I-24.

SANTOS, José (20I2). «Tiranía del paper. Imposición institucional de un tipo discursivo». Revista chilena de literatura 82, 197-217.

Sarlo, Beatriz (1996). «La duda y el pentimento». Punto de vista 56, 3I-35.

Schwarzböck, Silvia (2016). «De Bataille a Fogwill (o de la imaginación sadiana a la experiencia dictatorial)». El taco en la brea 3. En este mismo número.

Subercaseaux, Bernardo (2013). «Palabras del director». Revista Chilena de Literatura 84, 5-6.

Tejeda, Juan (2013). «La universidad nerd». Revista chilena de literatura 84, I55-160.

Tello, Maximiliano y Raúl Rodríguez Freire (Eds.) (20I2). Descampado. Ensayos sobre las contiendas universitarias. Santiago de Chile: Sangría.

Topuzián, Marcelo (2015). Creencia y acontecimiento. El sujeto después de la teoría. Buenos Aires: Prometeo.

Villalobos, Sergio (2013). «Revistas indexadas y otros vicios académicos». Revista chilena de literatura 84, $145-163$.

Wilkis, ARIel (20I5 [20I6]). «Sobre el capital moral. El itinerario de un concepto». El taco en la brea 3. En este mismo número. 


\section{Declaración sobre los índices de citación y las prácticas editoriales}

Por iniciativa de la Revista chilena de literatura, de la Universidad de Chile, los editores de algunas revistas latinoamericanas nos reunimos el 29 de septiembre del 2014 en Santiago de Chile para discutir políticas comunes y formas de apoyo en nuestra actividad. Los asistentes coincidimos en expresar nuestra inconformidad frente a las formas predominantes de medición de la calidad académica de las publicaciones que, en primer lugar, privilegian criterios administrativos y cuantitativos sobre los contenidos y, en segundo lugar, tienden a ignorar las prácticas académicas propias de las humanidades, que son diferentes a las de las ciencias exactas y aplicadas. Por eso, hemos decidido firmar la siguiente declaración pública, en cuya redacción hemos trabajado durante el primer semestre del 2015 .

\section{Antecedentes}

En los últimos ańos, varios gobiernos latinoamericanos han venido adoptando formas de medición de la calidad académica basadas en las nuevas políticas de administración pública, que privilegian el uso de indicadores y métricas por encima del contenido y del valor científico, social y cultural intrínseco del trabajo académico. Tales políticas han sido asumidas también por algunas universidades, cada vez más atentas a la visibilidad y el impacto, a la posición en los ránquines internacionales, y en general a la formación de capital humano en una perspectiva que privilegia el desarrollo económico.

Por lo general, los modelos de medición adoptados se basan en las prácticas académicas de las ciencias exactas y aplicadas, e ignoran las particularidades que caracterizan el trabajo académico en las ciencias humanas. Como criterio general, se suele privilegiar el paper como formato estándar de la producción académica, por encima de otras formas de difusión del conocimiento más afines a las humanidades, como el ensayo o el libro. Además, estos modelos conciben la utilidad del conocimiento de un modo restringido, limitado a la aplicación práctica y a la solución de problemas concretos.

Las ciencias humanas, por su naturaleza reflexiva y polémica, no se ajustan a este tipo de criterios, y esto no significa que sean menos importantes para la sociedad. El saber que ellas buscan es abierto y plural, no está dirigido exclusivamente a las comunidades académicas, sino también al ámbito público. Las humanidades fortalecen y alientan la apropiación crítica de la cultura y la tradición, abren espacios de discusión y debate, y tienen una dimensión utópica que va más allá de la mera solución de 
problemas inmediatos. Por eso, las humanidades no se adaptan fácilmente a los criterios meramente cuantitativos, ni a las formas estandarizadas de producción académica. De hecho, al adecuarse a los criterios de calidad imperantes, las humanidades a menudo se ven obligadas a traicionar su naturaleza, sus fines y su efecto social y cultural.

Las publicaciones que suscribimos el presente documento abogamos por una reformulación de los criterios de evaluación académica en las ciencias humanas. Nuestros comités editoriales comprenden la necesidad de la evaluación, pero se oponen a que ésta sea concebida a partir de principios cuantitativos o basados en la aplicación práctica inmediata del conocimiento. Dadas las diferencias de tradición e identidad entre las disciplinas, consideramos que tanto las universidades como los estados deben adoptar modelos de medición diferenciados, que tengan en cuenta las particularidades de cada una de ellas, y en cuya elaboración se cuente con una participación verdadera de las comunidades académicas. Sólo así podrán establecerse criterios claros para la adopción de políticas públicas con respecto a la investigación académica en nuestras áreas que redunden, efectivamente, en el bien general.

Algunos estados y universidades han adoptado, sin matices, criterios puramente cuantitativos de evaluación basados en los índices de citación, cuyos análisis y métricas se asumen como indicadores directos de la calidad de las publicaciones y de sus contenidos. La necesidad de publicar en revistas o en otros medios que se reportan en estos índices se ha convertido en política pública, en un imperativo para los investigadores, lo que afecta la lógica de la producción académica, los enfoques de las investigaciones, los formatos en los que se escribe y la naturaleza de algunos proyectos editoriales regionales. Esta exigencia y el enfoque cuantitativo dominante crean problemas para los in- vestigadores, y no sólo en el ámbito de las humanidades. En el área de las ciencias exactas y naturales han surgido voces críticas frente a los parámetros de evaluación y a la importancia excesiva que han adquirido los índices de citación y el factor impacto. La evaluación cuantitativa, han señalado, es apenas uno de los elementos de la evaluación de la calidad académica, pero no es el único, y ni siquiera el más importante. En todas las áreas, la evaluación académica debe ser contextual, pues debe hacerse a partir de la misión y el proyecto específico de las instituciones, de las publicaciones, de los distintos saberes disciplinarios, de los grupos de investigación y los individuos que son evaluados.

El contexto cultural y socioeconómico juega un papel importante en la consideración de la calidad de una publicación académica en cualquier área, pero especialmente en las humanidades y las ciencias sociales. Los indicadores suelen favorecer, por ejemplo, las publicaciones en inglés, pues tienen índices de citación más altos, se editan en países desarrollados y las más importantes se proponen como publicaciones «nucleares» (corejournals), es decir, publicaciones que consolidan un canon de la literatura académica más relevante para cada disciplina. Pero las ciencias humanas y sociales, por su propia naturaleza, están vinculadas a contextos regionales y lingüísticos específicos, y esos vínculos son fundamentales en la consideración de la calidad de los productos académicos. Así ha sido reconocido, por ejemplo, en el documento «Bases para la Categorización de Publicaciones Periódicas en Humanidades y Ciencias Sociales», publicado en junio del 2014 por el Consejo Nacional de Investigaciones Científicas y Técnicas (CONICET) de Argentina. Allí se establece con claridad que el factor de impacto no tiene la misma incidencia en las ciencias sociales y las humanidades que en otras disciplinas, y que los libros -individuales y colectivos - tienen una gran importancia en la producción cientí- 
fica de este campo, a pesar de que no suelan ser incorporados en los índices de citación. El documento dice, además, que criterios bibliométricos como el factor de impacto no deben ser usados sin más para evaluar la calidad de las publicaciones en ciencias sociales y humanidades. También vale la pena destacar la decisión del CONICET de poner en un mismo nivel los índices internacionales (wos, Scopus) y los regionales (scielo) o nacionales, como parte de una estrategia para fortalecer la producción regional, y para proponer la lengua española como un idioma de importancia en la generación de conocimiento y la difusión científica en las humanidades y ciencias sociales.

\section{Acuerdos para las prácticas editoriales y académicas}

Basados en los antecedentes anteriores, los comités editoriales de las revistas firmantes de la presente declaración hemos decidido formular una serie de acuerdos básicos que guíen nuestras prácticas editoriales y académicas:

- Consideramos que la calidad de nuestras revistas no se basa en un indicador de citación, sino en los contenidos que publican. Por eso, no utilizamos los índices de citación como herramienta promocional. La evaluación de los artículos recibidos tiene como criterios centrales la originalidad y la claridad de sus argumentos, y el aporte que ellos puedan hacer en la discusión académica sobre problemas literarios, estéticos, históricos y culturales. No se tienen en cuenta, por eso, aquellas cualidades o tendencias que puedan incidir directamente en el incremento de la citación de ningún autor o artículo, y mucho menos de cada una de nuestras revistas en su conjunto.

- Nuestras revistas promueven la lectura de sus contenidos y facilitan el acceso de los lectores, pero no obligan a los autores, por ejemplo, a citar artículos previamente publicados por ellas mismas, sino únicamente lo que sea relevante para los fines de cada texto, y de acuerdo con las recomendaciones que surjan del arbitraje por pares. Nuestras revistas tampoco se ciñen exclusivamente al formato del paper, ni a la estructura usual del artículo científico (introducción, métodos, resultados y discusión).

- Para nuestras revistas, los sistemas de indexación y resumen internacionales son un elemento clave en la difusión de sus contenidos, pues facilitan la localización de la información y el diálogo académico entre pares (esos eran, de hecho, sus propósitos iniciales). Sin embargo, una revista que no esté indexada en esos sistemas, especialmente en aquellos que miden la citación, no debería ser menos valorada por ese hecho.

- Nuestras revistas promueven la difusión gratuita de sus contenidos o su adquisición a precios asequibles para los lectores, pues consideramos que el conocimiento, el debate y la argumentación deben ser públicos.

- Nuestras revistas no cobran ni se proponen cobrar a los autores por publicar en ellas, para garantizar el acceso libre a sus contenidos.

Queremos invitar a otras revistas y editoriales académicas a suscribir la anterior declaración y a tener en cuenta los principios aquí establecidos. Hasta ahora, esta declaración tiene el respaldo de las siguientes publicaciones:

- Aletria, Revista de Estudos Literários (Universidade Federal de Minas Gerais, Brasil)

- ALPHA (Universidad de Los Lagos, Chile) 
- alter/nativas: revistas de estudios culturales latinoamericanos (Ohio State University, Estados Unidos)

- Ámbito de Encuentros (Universidad del Este, Puerto Rico)

- América, cabiers du Criccal (Université de Sorbonne-Nouvelle, Paris 3, Francia)

- Anclajes (Universidad Nacional de La Pampa, Argentina)

- Antares: Letras e Humanidades (Universidade de Caxias do Sul, Brasil)

- Anuario Colombiano de Historia Socialy de la Cultura (Universidad Nacional de Colombia)

- Artelogie (École des Hautes Études en Sciences Sociales, Francia)

- Babedec. Revista de Centro de Estudios de Teoría y Crítica Literaria (Universidad Nacional de Rosario, Argentina)

- Belas Infiéis (Universidade de Brasilia, Brasil)

- Biotecnología en el sector agropecuario y agroindustrial (Universidad del Cauca, Colombia)

- Bitácora urbano/territorial (Universidad Nacional de Colombia)

- Boletín de arqueología (Pontificia Universidad Católica del Perú)

- Boletín cientifico (Universidad de Caldas, Colombia)

- Boletín del Centro de Estudios de Teoría y Crítica Literaria (Universidad Nacional de Rosario, Argentina)

- Boletín de filología (Universidad de Chile)

- Brumal. Revista de Investigación sobre lo fantástico (Universidad Autónoma de Barcelona)

- CAFE. Cahiers des Amériques, Figures de l'Entre (Université de La Rochelle, Francia)

- Caracteres. Estudios culturales y críticos de la esfera digital (Universidad de Salamanca, España)

- Caribe: revista de cultura y literatura (Marquette University, Estados Unidos)

- CELEHIS (Universidad Nacional de Mar del Plata, Argentina)

- Centroamericana (Università Cattolica del Sacro Cuore, Milano, Italia)

- Colindancias (Universidad de Oeste de Timisoara, Rumania)

- Cuadernos del CILHA (Universidad Nacional de Cuyo, Argentina)

- Cuadernos de historia (Universidad de Chile)

- Cultura del cuidado enfermería (Universidad Libre, Colombia)

- Decimonónica: revista de producción cultural hispánica decimonónica (Estados Unidos)

- Desafios (Universidad del Rosario, Colombia)

- Desde el jardín de Freud. Revista de psicoanálisis (Universidad Nacional de Colombia)

- Diálogos de Saberes. Investigaciones en Derecho y Ciencias Sociales (Universidad Libre, Colombia)

- Dirāsāt Hispannicas. Revista Tunecina de Estudios Hispánicos (Universidad el Manar, Túnez)

- Editorial Jorge Millas (Fundación Jorge Millas, Chile)

- El Ágora USB (Universidad San Buenaventura, Medellín, Colombia) 
- El taco en la brea (Universidad Nacional del Litoral, Argentina)

- Encuentros (Universidad Autónoma del Caribe, Colombia)

- Entramado (Universidad Libre, Sede Cali, Colombia)

- Episteme. Revista de Ciencias Sociales y Humanas (Universidad Santo Tomás, Sede Villavicencio, Colombia)

- e-scrita (Uniabeu, Brasil)

- Estudios. Revista de Investigaciones Literarias y Culturales (Universidad Simón Bolívar, Venezuela)

- Estudios Avanzados. Instituto de Estudios Avanzados (Universidad de Santiago de Chile)

- Estudios de Filosofía (Universidad de Antioquia, Colombia)

- Estudios de Literatura Colombiana (Universidad de Antioquia, Colombia)

- Estudos de Literatura Brasileira Contemporânea (Universidade de Brasilia, Brasil)

- Folia Histórica del Nordeste (Universidad Nacional del Nordeste, Argentina)

- Fronteras de la historia (Instituto Colombiano de Antropología e Historia, Colombia)

- Gramma (Universidad del Salvador, Argentina)

- Hispamérica (University of Maryland, Estados Unidos)

- Historia Caribe (Universidad del Atlántico, Colombia)

- HOW (Asociación Colombiana de Profesores de Inglés, Colombia)

- Humanidades (Universidad de Montevideo, Uruguay)

- Hypnos (Pontifícia Universidade Católica de São Paulo, Brasil)

- Ideas y Valores. Revista Colombiana de Filosofía (Universidad Nacional de Colombia)

- Izquierdas (Instituto de Estudios Avanzados, Universidad de Santiago de Chile)

- Katatay. Revista critica de literatura latinoamericana (Red Interuniversitaria Katatay, Argentina)

- Kavilando. Revista de ciencias sociales y humanas (Grupo de Investigación para la Transformación Social Kavilando, Colombia)

- Kaypunku, revista de Estudios Interdisciplinarios de Arte y Cultura (Grupo de Investigación Kaypunku, Perú)

- Kipus: Revista andina de letras (Universidad Andina Simón Bolívar, Ecuador)

- La manzana de la discordia (Universidad del Valle, Colombia)

- La Palabra (Universidad Pedagógica y Tecnológica de Colombia)

- Lenguaje (Universidad del Valle, Colombia)

- Letras (Universidad de San Marcos, Perú)

- Lexis. Revista de lingüistica y literatura (Universidad Católica del Perú)

- Linguas \& Letras (Universidade Estadual do Oeste do Paraná, Brasil)

- Lingüistica y Literatura (Universidad de Antioquia, Colombia)

- Literatura: teoría, historia, crítica (Universidad Nacional de Colombia)

- Luciérnaga (Politécnico Colombiano Jaime Isaza Cadavid, Colombia)

- Lúmina (Universidad de Manizales, Colombia)

- Memoria y sociedad (Pontificia Universidad Javeriana, Colombia)

- Milenio. Revista de Artes y Ciencias (Universidad de Puerto Rico, sede Bayamón) 
- Mora (Universidad de Buenos Aires, Argentina)

- Mundo amazónico (Instituto Imani, Universidad Nacional de Colombia, Sede Amazonia)

- Olho d'água (Universidade Estadual Paulista «úlio de Mesquita Filho», Brasil)

- Olivar: revista de literatura y cultura españolas (Universidad Nacional de La Plata, Argentina)

- Orbis Tertius (Universidad Nacional de La Plata, Argentina)

- Perifrasis. Revista de Literatura, Teoría y Crítica (Universidad de los Andes, Colombia)

- Perspectiva geográfica (Universidad Pedagógica y Tecnológica de Colombia Instituto Geográfico Agustín Codazzi, Colombia)

- Pilquen. Sección Ciencias Sociales (Universidad Nacional del Comahue, Centro Universitario Zona Atlántica, Argentina)

- Pilquen. Sección Psicopedagogía (Universidad Nacional del Comahue, Centro Universitario Zona Atlántica, Argentina)

- Polifonía. Revista de Estudios Hispánicos (University of San Francisco, Estados Unidos)

- Praesentia, revista venezolana de estudios clásicos (Universidad de Los Andes, Venezuela)

- Prismas. Revista de historia intelectual (Universidad Nacional de Quilmes, Argentina)

- PROFILE Issues in Teachers' Professional Development (Universidad Nacional de Colombia)

- Psicoespacios (Institución Universitaria de Envigado, Colombia)

- Quipu, Revista Latinoamericana de Historia de las Ciencias y la Tecnología (Comunidad en Internet de Historia de la Ciencia y la Tecnología en América Latina, México)

- Rastros Rostros (Universidad Cooperativa de Colombia)

- Recial (Universidad Nacional de Córdoba, Argentina)

- Revista Archivos de medicina (Universidad de Manizales, Colombia)

- Revista colombiana de gastroenterología (Asociación Colombiana de Gastroenterología, Colombia)

- Revista Colombiana de Sociología (Universidad Nacional de Colombia)

- Revista chilena de literatura (Universidad de Chile)

- Revista de Filosofía (Universidad de Chile)

- Revista de la Asociación Colombiana de Ciencias Biológicas (Asociación Colombiana de Ciencias Biológicas, Colombia)

- Revista de Literaturas Modernas (Universidad Nacional de Cuyo, Argentina)

- Revista de psicología GEPU (Universidad del Valle, Colombia)

- Revista Historia y Sociedad (Universidad Nacional de Colombia, Sede Medellín)

- Revista Iberoamericana (Instituto Internacional de Literatura Iberoamericana, Universidad de Pittsburgh, Estados Unidos)

- Revista INNOVAR (Universidad Nacional de Colombia) 
- Revista Instituto Colombiano de Derecho Tributario (Instituto Colombiano de Derecho Tributario, Colombia)

- Revista Laboratorio (Universidad Diego Portales, Chile)

- Revista latinoamericana de etnomatemática (Universidad de Nariño, Colombia)

- Revista Lebret (Universidad Santo Tomás, Colombia)

- Revista Miradas (Universidad Tecnológica de Pereira, Colombia)

- Revista Nomadias (Universidad de Chile)

- Revista Poiésis (Fundación Universitaria Luis Amigó, Colombia)

- Revista U.D.C.A Actualidad \& Divulgación Cientifica (Universidad de Ciencias Aplicadas y Ambientales, Colombia)

- RIVAR, Revista Iberoamericana de Viticultura, Agroindustria y Ruralidad (Instituto de Estudios Avanzados, Universidad de Santiago de Chile)

- Tejuelo. Didáctica de la Lengua y la Literatura (Universidad de Extremadura, España)

- Telar (Universidad Nacional de Tucumán, Argentina)

- Tendencias \& Retos (Universidad de La Salle, Colombia)

- Teuken Bidikay (Politécnico Colombiano Jaime Isaza Cadavid, Colombia)

- Trabajo Social (Universidad Nacional de Colombia)

- Verba Hispánica (Universidad de Ljubljana, Eslovenia)

- Zama (Instituto de Literatura Hispanoamericana de la Facultad de Filosofía y Letras, Universidad de Buenos Aires, Argentina) 